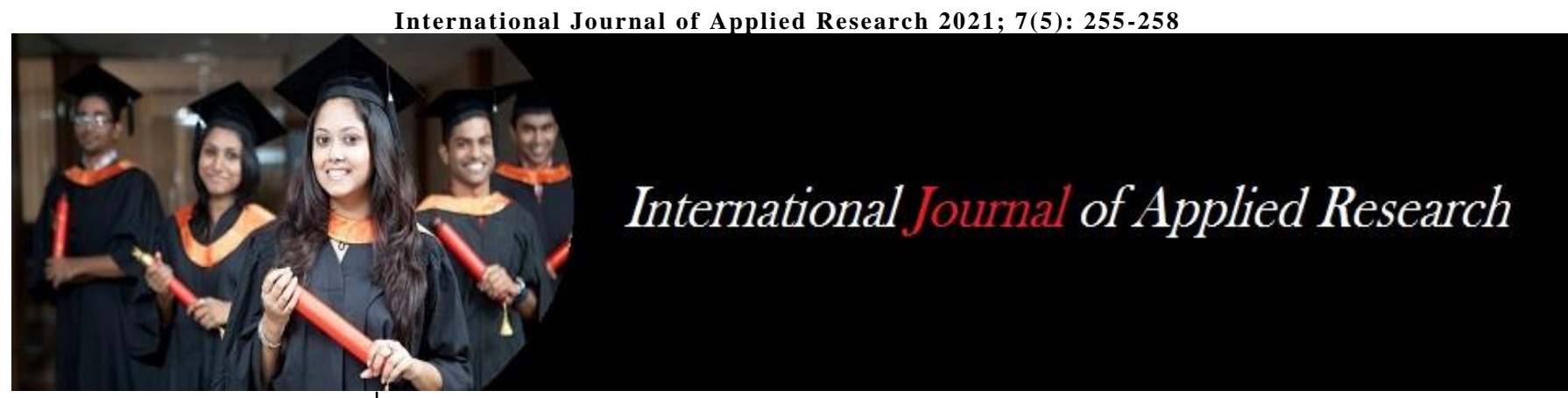

ISSN Print: 2394-7500

ISSN Online: 2394-5869

Impact Factor: 8.4

IJAR 2021; 7(5): 255-258

www.allresearchjournal.com

Received: 25-03-2021

Accepted: 27-04-2021

Dr. Vanisha Steven

Dr. D.Y. Patil College of

Physiotherapy, Pimpri, Pune,

Maharashtra, India

Dr. Roopa Desai

Dr. D.Y. Patil College of

Physiotherapy, Pimpri, Pune,

Maharashtra, India

Dr. Preeti Gazba

Dr. D.Y. Patil College of

Physiotherapy, Pimpri, Pune,

Maharashtra, India

Dr. Shilpa Khandare

Dr. D.Y. Patil College of

Physiotherapy, Pimpri, Pune,

Maharashtra, India

\section{Scapula dyskinesia in hospital housekeepers: A prevalence study}

\section{Dr. Vanisha Steven, Dr. Roopa Desai, Dr. Preeti Gazbare and Dr. Shilpa Khandare}

DOI: https://doi.org/10.22271/allresearch.2021.v7.i5d.8579

\section{Abstract}

Background: Hospital Housekeeping staff perform repetitive and forceful upper limb motion which gives rise to scapula muscle fatigue which lead to muscle weakness and alters the position and motion of the scapula. These alterations in the scapula predispose the housekeepers to various work related disorders and injuries.

Objective: To determine the prevalence of Scapula Dyskinesia in Hospital Housekeeping staff.

Methods: A total of 134 hospital housekeeping staff were enrolled in the study. Participants were evaluated for work related musculoskeletal pain using a self formulated questionnaire. Scapula dykinesia was assessed in both static position and during dynamic scapula motion. Observation of the scapula at rest was made to determine the alteration in the static position of the scapula, the Lateral Scapula Slide Test assessed at three different positions was used to assess the dynamic scapula motion. Result: $94.77 \%$ of the participants reported to have work related musculoskeletal pain. Majority of participants experienced pain in the low back region followed by pain in shoulder, neck and knee region. Scapula dyskinesia was observed in 30(22.38\%) of 134 participants. The mean values of LSST for position 1 was $7.91 \pm 1.09$, position 2 was $8.06 \pm 1.07$, position 3 was $7.62 \pm 1.05$.

Conclusion: A large number of housekeeping staff suffer from work related musculoskeletal pain, among them $22.38 \%$ of the hospital housekeeping staff observed to have Scapula Dyskinesia.

Clinical Implication: Scapula Dyskinesia leads to biomechanical alterations in the individuals and predispose them to suffer from various work related musculoskeletal disorders,' which may cause work related disability, decrease in work productivity and reduced quality of life in these housekeeping staff.

Keywords: lateral scapula slide test, housekeepers, work related musculokeletal disorders

\section{Introduction}

Scapula dyskinesia is the visible alterations in the normal position or motions of the scapula occurring during scapular and humeral movements. 'Dyskinesis' implies to the loss of normal control of scapular motion. Various bones, soft tissue and nerves related factors around the scapula may contribute to scapula dykinesia. Among which reduced mobility of the scapula muscle, distorted muscle activation patterns and altered scapula muscle force couple coactivation are the soft tissue related causes which lead to the occurrence of scapula dyskinesia ${ }^{[1]}$ Most commonly the lower stabilizers of the scapula which include the serratus anterior, rhomboids, middle and lower trapezius muscles are inhibited or weakened in scapula dyskinesia ${ }^{[2]}$ Inhibition of these muscles decreases the ability of the muscles to exert torque and stabilize the scapula, as well as alter the normal firing patterns of the muscles around the shoulder. An altered scapular biomechanics that occur as a result of dysfunction create abnormal scapular positions that reduces the normal shoulder function and predispose the shoulder to injury ${ }^{[3,4]}$. Housekeeping department play a major role in hospitals inorder to maintain a clean health care environment. Housekeeping staff are primary and vital components of the housekeeping department, the individuals working in the housekeeping department are generally involved in physically active jobs and perform variety of movements such as walking, frequent forward bending, twisting motions of trunk, stooping, crouching and kneeling. Furthermore since these housekeepers repeatedly perform physically hard and tiring work such as mopping, vaccuming, moving objects, polishing floors, cleaning 
walls and ceilings, their job is classified as medium to heavy work ${ }^{[5]}$. Majorly the housekeeping job include task which necessitate performance of repeated upper extremity activities, a large amount of which involve performing those activities above their shoulder level such as while performing high pressure spraying or overhead cleaning, these repeated overhead activities impart high physical load to the upper extremity of these individuals. Repetitive upper extremity motion, heavy work loads, poor deign of the cleaning tools, meagre work organization may all lead to scapula muscle fatigue. Scapula muscle fatigue in housekeepers in turn alters the scapulo-humeral rhythm, resulting in abnormal compensatory motions of the scapula or destabilization of the scapula. Scapula dyskinesia thus increase the risk of work related musculoskeletal disorders or injuries in these housekeeping staff ${ }^{[6]}$. Previous studies reported the prevalence of scapula dyskinesia to be $61 \%$ in overhead and $33 \%$ in non overhead athletes. However there is a dearth of literature on scapula dyskinesia in housekeepers, this study therefore aims to determine the prevalence of Scapula dyskinesia in Hospital Housekeeping Staff.

\section{Methodology \\ Study design: An Observational study}

Sampling technique: Simple random sampling

\section{Sample size: 134}

Study duration: After obtaining an ethical clearance from the Institutional Ethical Committee of Dr. D.Y. Patil Vidyapeeth Pune, India, the study was conducted between June 2019 to January 2020

\section{Selection criteria}

Inclusion criteria: Hospital Housekeeping staff of Dr. D.Y. Patil Vidyapeeth campus Pune, India in the age group of 18 -50 years, both males and females, and those willing to participate in the study.

Exclusion criteria: Participant with congenital or traumatic shoulder conditions, recent history of upper extremity injury or surgeries and neurological disorders that affect muscle strength were excluded from the study.

Methodology: 134 hospital housekeeping staff were included in the study. A verbal and written informed consent in the local language was taken from the participants before the commencement of the study. The Principal Investigator (Trained Physiotherapist) undertook a standardized history and physical examination of all the participants. The self prepared Questionnaire was validated by the staffs who were experienced in the field. A structured self made questionnaire formulated by the principal investigator was used to collect the relevant information from the participants. The questionnaire included the following information 1) Demographic data 2) Questions pertaining to their job. The demographic data included the name, age, gender, address, contact details and work experience of the participants. Work related questions included- the duration of the job activity, activities maximally involved in the job, total number and duration of breaks, presence of work related symptoms such as pain, site and duration of pain, and whether their work is hampered due to pain were taken. The participants were screened for scapula dyskinesia with scapula in both static and dynamic position. Static assessment involved observation of the scapula in static position to determine the orientation of scapula at rest. Dynamic scapula motion was evaluated using the Lateral Scapula Slide Test (LSST), which determines the stability of the scapula during glenohumeral movement. For this test, the participants were asked to adequately expose the scapula to allow accurate readings, the participants were asked to stand with their arms at the side of the body, the distance from the inferior angle of the scapula to the spinous process of $\mathrm{T} 7$ or T9 was measured with the help of a measuring tape. The particapants were then tested using two other positions at $45^{\circ}$ shoulder abduction(hands on waist with thumbs posteriorly) and $90^{\circ}$ shoulder abduction with internal rotation, if the measured distance between the right and left side was more than $1.5 \mathrm{~cm}$ the test was considered to be positive for scapula dyskinesia ${ }^{[7]}$.

\section{Results}

The data was analysed using descriptive statisctics. The study included 134 hospital housekeeping staff, 132 females and 2 males. The mean age group of the participants was $35.76 \pm 6.38$. The total job duration for all the participants was more than 8 hours per day, with a single break period of one hour. All 134 participants had to work with upper extremities in various position a such as working at, above or below the shoulder or at the waist level. Out of the total, 127 participants reported to have work related musculoskeletal pain and also reported to have difficulty in performing their daily activities due to pain which in turn hampered their work performance. Pain was experienced at one or more at the following body sites, low back (49\%), shoulder (47\%), neck (44\%), knee (27\%), upper back $(2 \%)$, wrist $(1 \%)$. The mean value of pain at rest was $2.70 \pm 1.50$ and on activity was $5.96 \pm 1.32$. Among the participants experiencing pain, 101 participants experienced pain for more than 6months, 31 participants had pain for less than 6 months and 5 participants experienced pain for less than 1 month duration. Assessment of scapula in static position revealed that 103 Participants had normal scapula position, 21 had inferior medial border prominence, 7 participants showed prominence of the entire medial border, elevated scapula was reported in only 1 participant and 1 participant was revealed to have an abducted scapula in static position. 30 female participants of $134(22.38 \%)$ participants were tested positive on the LSST and reported to have Scapula Dyskinesia. All most all (96.66\%) the participants with scapula dyskinesia had an affection of the dominant extremity, the right scapula was affected in 28 participants, whereas 2 participants had an affection of the left side. All the participants with scapula dyskinesia reported to have maximal amount of pain in either the shoulder or neck region.

Table 1: Demographic data of the participants

\begin{tabular}{|c|c|}
\hline Characteristics & Mean Value \\
\hline Age & $35.76 \pm 6.38$ \\
\hline Males & 2 \\
\hline Females & 132 \\
\hline Work experience & $2.70 \pm 1.35$ \\
\hline Right hand dominant & 129 \\
\hline Left hand dominant & 5 \\
\hline
\end{tabular}




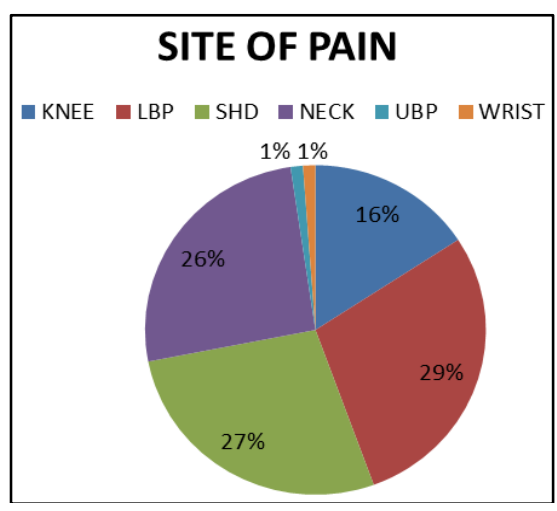

Fig 1: Describing the site of pain
Interpretation- Out of the total, $29 \%$ of participants demonstrated to have pain in the low back region, $27 \%$ had shoulder pain, $26 \%$ had neck pain, $16 \%$ reported to have pain in the knee region, whereas only $1 \%$ (in each) of the participants suffered from upper back and wrist pain.

Table 2: NPRS on rest and on activity

\begin{tabular}{|c|c|c|}
\hline Pain on NPRS & Mean value & SD \\
\hline Rest & 2.7 & 1.50 \\
\hline Activity & 5.98 & 1.32 \\
\hline
\end{tabular}

Interpretation-table 1 shows mean value of NPRS on pain on rest was $2.7 \pm 1.50$ and on activity was $5.98+1.32$

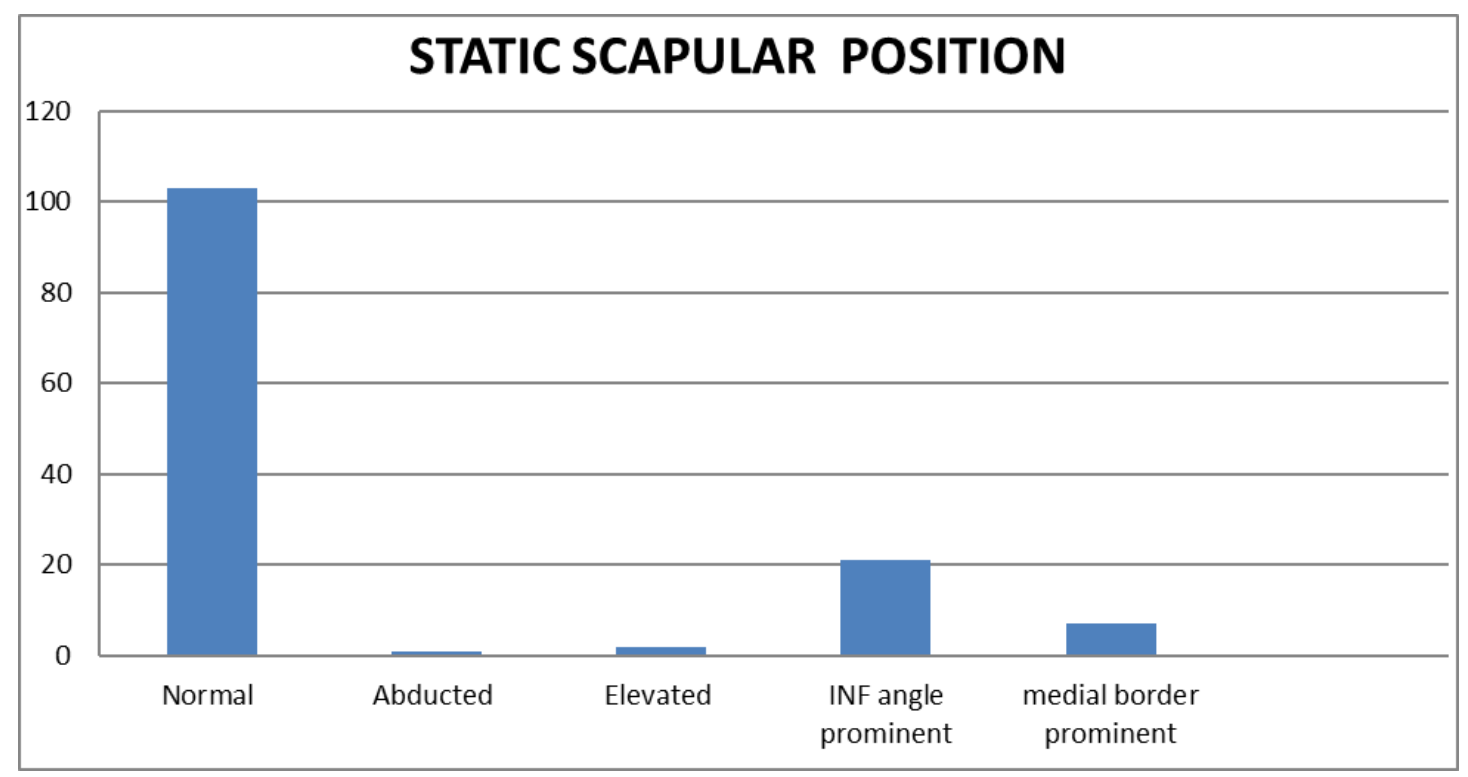

Fig 2: Graphical representation of the scapula in Static position

Interpretation-103 Participants had normal scapula position, 21 participants had inferior angle prominence, the medial border was prominent in 7 participants, elevated scapular position was reported in 2 , and an abducted position of the scapula was observed in only 1 participant.

Table 3: LSST on right and left side at 3 different position

\begin{tabular}{|c|c|c|c|c|}
\hline Lsst & \multicolumn{2}{|c|}{ Right Side } & \multicolumn{2}{c|}{ Left Side } \\
\hline & Mean & SD & Mean & SD \\
\hline Position 1 & 7.91 & 1.09 & 7.49 & 0.81 \\
\hline Position 2 & 8.06 & 1.07 & 7.60 & 0.81 \\
\hline Position 3 & 7.62 & 1.05 & 7.15 & 0.79 \\
\hline
\end{tabular}

Interpretation-table 2 shows mean values of LSST on right and left side at 3 different positions, the mean value for LSST for position 1(arms at the side) was $7.91 \pm 1.09$ on the right and $7.49 \pm 0.81$ on the left, mean value for position 2 ( $45^{\circ}$ shoulder abduction) was $8.06 \pm 1.07$ and $7.60 \pm 0.81$ on the right and left side respectively, mean value for position $3\left(90^{\circ}\right.$ shoulder abduction with internal rotation) on the right was $7.62 \pm 1.05$ and left was $7.15 \pm 0.79$

\section{Discussion}

The study included 134 hospital housekeeping staff, with the mean age of $35.76 \pm 6.38$, the study revealed that maximum number $(94.77 \%)$ of the participants suffered work related musculoskeletal pain with the highest magnitude of pain was experienced in the low back, shoulder, neck and knee region, least amount of pain were reported in other body parts such as the upper back and wrist.

All the participants included in the study experiencing pain in any of the body areas also reported to have difficulty in performing work related task, which also hampered their job performance. The musculoskeletal symptoms in these individuals can be explained by the nature of the job they are involved in, which places a large amount of physical load on their musculoskeletal system. 30(22.38\%) of 134 participants included in the study suffered from scapula dyskinesia, which was reported by either the inferio medial border prominence $(70 \%)$ or prominence in the entire medial border of the scapula $(23.33 \%)$ assessed with the scapula in the static position, and a positive LSST. Among the 30 participants with scapula dyskinesia, right scapula was affected in 28 participants, whereas 2 participants had an affection of the left scapula. All the participants with scapula dyskinesia reported to have maximal amount of pain in either the shoulder or neck region. Scapula Dyskinesia in these housekeeping staff may be due to the nature of their job which involve performing various activities such as cleaning, washing, lifting, pulling and carrying heavy loads, working with arms above the shoulder, and work in a range of awkward posture, also the repeated upper extremity activities performed by them, may contribute lead to fatigue in the muscles of the rotator cuff and the scapula stabilizers (upper-middle-lower trapezius, serratus anterior and the 
latissimus dorsi), fatigue in these muscles in turn induces weakness in these musculature. Fatigue alters the timing of the scapula humeral rhythm, and may have a effect on the scapulo-humeral and scapula thoracic range of motion. Therefore as housekeepers usually work in posture which are unnatural and deviate from the normal, these positions place greater amount of stress on the surrounding soft tissues and joints and cause biomechanical alterations in the individuals, predisposing them to suffer from various work related musculoskeletal disorders ${ }^{[7]}$. These occupation related musculoskeletal disorders are the second largest cause of temporary work disability and are widely associated with huge expenses of health cost, reduction in work productivity and reduced quality of life in these workers ${ }^{[8]}$

Future scope-Further studies can be conducted using a larger study population. The Lateral Scapula Slide Test can be performed using a vernier calliper to improve the accuracy of the readings obtained.

\section{Conclusion}

The study revealed that a large number of housekeeping staff suffer from work related musculoskeletal pain, among them $22.38 \%$ of the hospital housekeeping staff observed to have Scapula Dyskinesia.

Conflict of interest: The authors declare no conflict of interest with results of this study

Source of funding: The study was self funded

\section{Acknowledgement}

We express our gratitude towards the staff of the Housekeeping Department of Dr. D.Y Patil Vidyapeeth campus Pune for their constant support provided for the completion of the study.

\section{References}

1. Peat M. Functional anatomy of the shoulder complex. Physical therapy 1986;66(12):1855-65.

2. DiGiovine NM, Jobe FW, Pink M, Perry J. An electromyographic analysis of the upper extremity in pitching. Journal of shoulder and elbow surgery 1992;1(1):15-25.

3. Ben Kibler W. The role of the scapula in athletic shoulder function. The American journal of sports medicine 1998;26(2):325-37.

4. Kibler BW, Sciascia A, Wilkes T. Scapular dyskinesis and its relation to shoulder injury. JAAOS-journal of the American academy of orthopaedic surgeons 2012;20(6):364-72.

5. Coşkuner S, Hazer O. Risk Factors for Housekeeping Employees in their Work Areas and the Ergonomic Solutions. International Journal of the Humanities 2009;15;6(12).

6. Parmar S, Dalal P. A study of musculoskeletal disorder among housekeeping staff in hotel industry. Int J Home Sci 2017;3(3):83-5.

7. David J Magee. Orthopedic Physical Assessment. $6^{\text {th }}$ ed, Saunders, St Louis Missouri 2014, 252-387.

8. Karwowski W, Marras WS, editors. Occupational ergonomics: principles of work design. CRC press, 2003, 26.
9. Wami SD, Dessie A, Chercos DH. The impact of workrelated risk factors on the development of neck and upper limb pain among low wage hotel housekeepers in Gondar town, Northwest Ethiopia: institution-based cross-sectional study. Environmental health and preventive medicine 2019;24(1):1-0. 